\title{
IgD multiple myeloma with central nervous system involvement: A case report and literature review
}

\author{
YI CHEN, YANYAN QIU, HAIYING FU, JING LI, LUSHAN CHEN, SIQIN LIAO and TINGBO LIU \\ Fujian Provincial Key Laboratory on Haematology, Fujian Medical University Union Hospital, \\ Fujian Institute of Haematology, Fuzhou, Fujian 350001, P.R. China
}

Received September 16, 2020; Accepted March 17, 2021

DOI: $10.3892 / \mathrm{mco} .2021 .2360$

\begin{abstract}
Central nervous system (CNS) involvement is a rare manifestation of multiple myeloma (MM) and the optimal management strategies have yet to be determined. The aim of the present study was to describe the case of a 47-year-old male patient with immunoglobulin D- $\lambda$ MM who presented with multiple extramedullary infiltrations at diagnosis. This patient achieved stringent complete response after 9 cycles of treatment with bortezomib, lenalidomide and dexamethasone, and then received lenalidomide as maintenance therapy. CNS involvement and extramedullary relapse developed 3 months after the last chemotherapy cycle. Despite receiving a second-line treatment protocol, the patient succumbed to the disease within 1 month after recurrence. The characteristics and treatment options for CNS MM are discussed in this case report.
\end{abstract}

\section{Introduction}

Multiple myeloma (MM) is characterized by clonal proliferation of plasma cells in the bone marrow. Extramedullary plasmacytomas (EMPs) may also develop, and they may either be present at diagnosis or develop later during the disease course (1). EMP has a cumulative incidence of $9 \%$ among all patients with MM and is usually associated with a poor prognosis (2,3). Serum monoclonal immunoglobulin (Ig) allows distinguishing between different types of MM, and a multicentre analysis reported that IgD-subtype myeloma accounts for $6.5 \%$ of all cases in China (4). IgD MM presenting with muscle infiltration at diagnosis and central nervous system (CNS) involvement after complete response (CR) are rare occurrences. Due to the rarity of this subtype and incomplete

Correspondence to: Dr Tingbo Liu, Fujian Provincial Key Laboratory on Haematology, Fujian Medical University Union Hospital, Fujian Institute of Haematology, 29 Xinquan Road, Fuzhou, Fujian 350001, P.R. China

E-mail: liutb668@139.com

Key words: multiple myeloma, extramedullary, central nervous system, involvement understanding of its clinical course, the optimal diagnostic and management strategies have yet to be determined.

In the present study, a case of IgD- $\lambda$ MM with CNS involvement was retrospectively analyzed and a systematic review of the literature was performed to provide a contemporary update on the incidence, demographics, management and outcome of EMP with CNS involvement.

\section{Case report}

A 47-year-old male patient was admitted to the Putian Hospital (Putian, China) in March 2019 with a chief complaint of blepharoptosis for 4 months and fatigue for 2 months. There were no other complaints, and the routine blood tests were normal. An enhanced magnetic resonance imaging (MRI) brain scan performed in March 2019 revealed soft tissue masses in the sphenoid and bilateral cavernous sinuses, and further examinations were recommended. Positron emission tomography/computer tomography (PET-CT) performed in April 2019 revealed abnormal bone density of the whole body, multiple soft tissue masses, and a pathological fracture of the 10th rib on the left side (Fig. 1). Biopsy of the chest wall mass was performed in April 2019, and examination of the bioptic material revealed abnormal plasma cell infiltration of muscle tissue, after which time the patient was referred to the Fujian Medical University Union Hospital (Fuzhou, China) for further investigation and treatment.

Following admission in April 2019, further evaluations were performed. Blood and biochemistry tests revealed normal levels of haemoglobin, calcium, lactate dehydrogenase, albumin, globulin, creatinine and serum $\beta 2$-microglobulin (Table I). Serum immunofixation electrophoresis indicated IgD- $\lambda$ light chains, and the levels of serum free $\lambda$ and $\kappa$ light chains were 151.0 and $14.3 \mathrm{mg} / \mathrm{l}$, respectively. Bone marrow aspiration revealed that the proportion of plasma cells was $32.0 \%$, as shown in Fig. 2. Flow cytometry revealed abnormal clonal plasma cells that expressed CD38, CD138 and $\lambda$ light chains, and were negative for CD19, CD56, CD117 and $\kappa$ light chains (Fig. 3). A large number of immature plasma cells were identified on bone marrow biopsy. The results of immunohistochemistry (Fig. 4) were as follows: CD38 (3+), CD138 (3+), $\lambda(3+)$, CD56 (-), CD20 ( \pm ) and $\kappa(-)$. Furthermore, the biopsy of the chest wall mass was reviewed, and a mass of immature plasma cells was identified in the muscle tissue. 
Table I. Biochemical parameters at diagnosis and at the time of recurrence.

\begin{tabular}{lcc}
\hline Parameters & Diagnosis & Recurrence \\
\hline Leukocyte count $\left(4-10 \times 10^{9} / \mathrm{l}\right)$ & 4.9 & 7.5 \\
Haemoglobin $(\mathrm{g} / \mathrm{l})$ & 134 & 107 \\
Platelet count $\left(100-300 \times 10^{9} / \mathrm{l}\right)$ & 142 & 155 \\
Immunoglobulin $(20-35 \mathrm{~g} / \mathrm{l})$ & 27.7 & 21.8 \\
Albumin $(35-54 \mathrm{~g} / \mathrm{l})$ & 42 & 43 \\
Creatinine $(40-135 \mu \mathrm{mol} / \mathrm{l})$ & 65 & 78 \\
Calcium $(2.1-2.7 \mu$ mol/l) & 2.34 & 2.2 \\
Lactate dehydrogenase $(109-245 \mathrm{IU} / \mathrm{l})$ & 208 & 309 \\
Serum $\beta 2$-microglobulin $(1.09-2.53 \mathrm{mg} / \mathrm{l})$ & 2.5 & 2.37 \\
Bone marrow plasma cell $(\%)$ & 32 & 1 \\
Chromosome & $46, \mathrm{XY}$ & - \\
MM fluorescence in situ hybridization & Negative & -
\end{tabular}
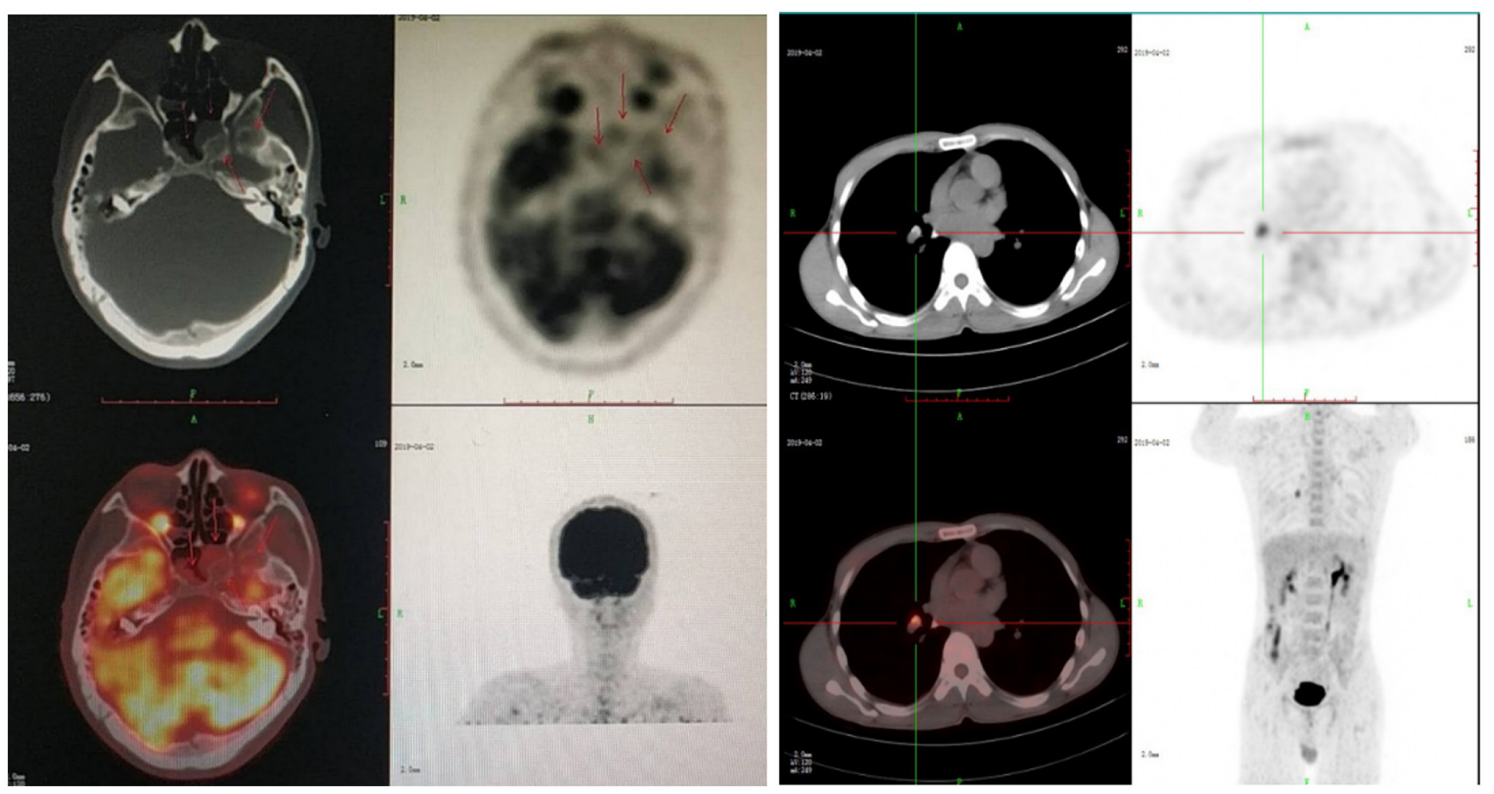

Figure 1. Baseline positron emission tomography-computed tomography scan. Abnormal bone density of the whole body, multiple soft tissue masses (arrows), and a pathological fracture of the 10th rib on the left side were observed.

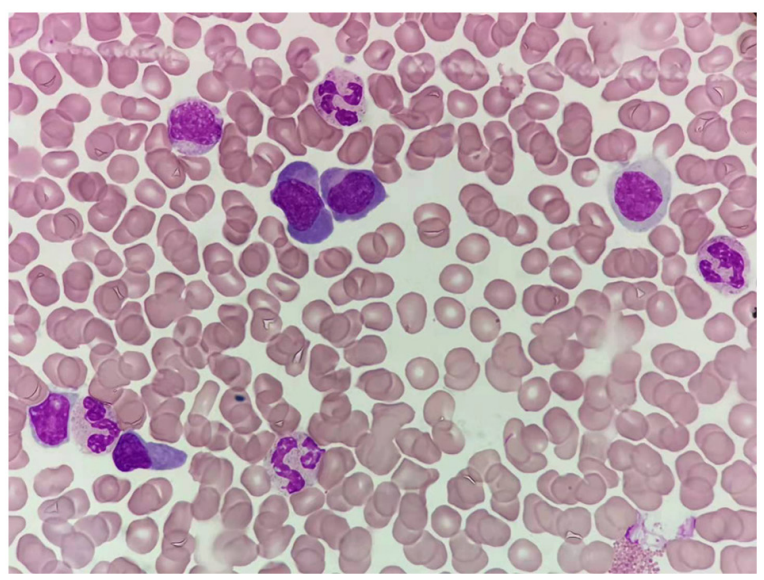

Figure 2. Baseline bone marrow aspiration. On bone marrow examination, the proportion of plasma cells was $32.0 \%$ (Wright's stain; magnification, $\mathrm{x} 100)$.
The following antibodies were ready to use and applied on the Lumatas automated immunostainer (Fuzhou Maixin Biotech. Co., Ltd.) with EDTA heat retrieval solution (Fuzhou Maixin Biotech. Co., Ltd.): Anti-CD20, anti-CD79 $\alpha$, anti-CD138, anti- $\kappa$, anti- $\lambda$ and anti-multiple myeloma oncogene (MUM)1, and the details are listed in Table II. Staining intensity level was defined as follows: -, not stained; +, weakly stained; ++, moderately stained; and +++, strongly stained. Flow cytometry was performed with BD FACSCanto (Becton, Dickinson and Company). The results of immunohistochemistry (Fig. 5) were as follows: CD38 (3+), CD138 (3+), $\lambda(3+), \kappa( \pm)$, MUM1 (2+), CD56 (-), CD20 (-), Pax5 (-) and CD79a ( \pm ). The results of the chromosome and $\mathrm{MM}$ fluorescence in situ hybridization assays were normal.

Based on the aforementioned findings, a diagnosis of active MM with multiple extramedullary foci, Revised International Staging System stage I, low-risk group, was confirmed 

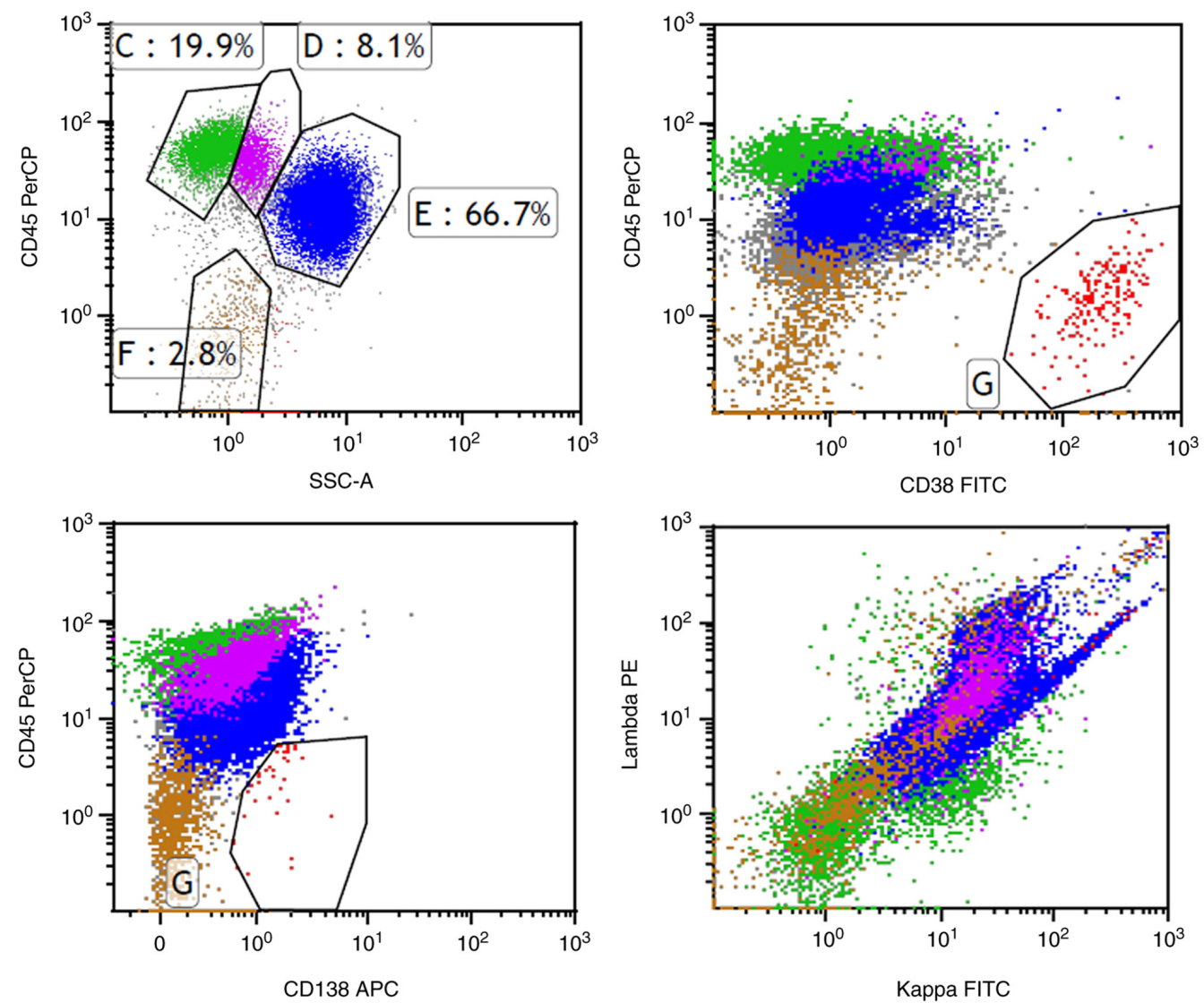

Figure 3. Baseline flow cytometry of bone marrow. The plasma cells expressed CD38, CD138, and $\lambda$ light chains and were negative for CD19, CD56, CD117 and $\kappa$ light chains.

A

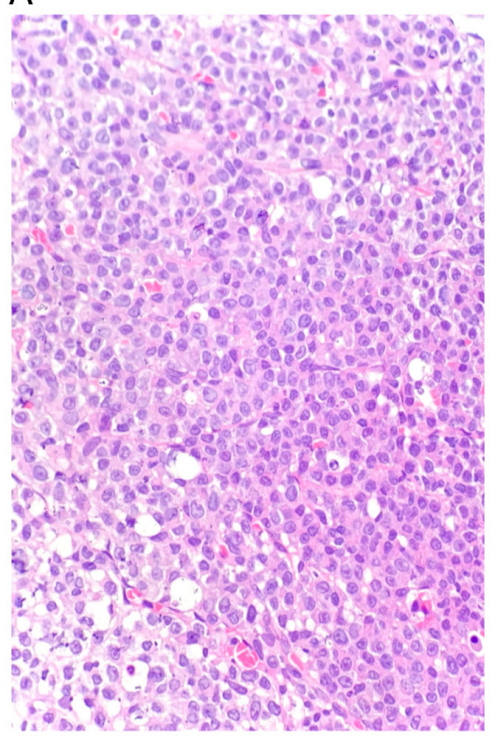

B

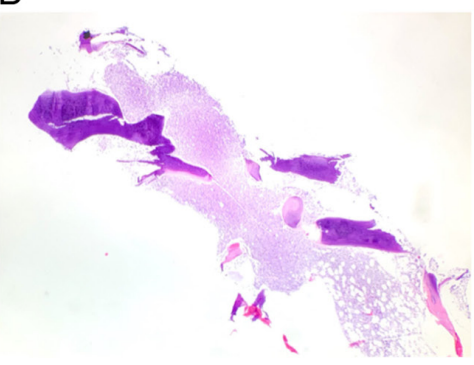

D

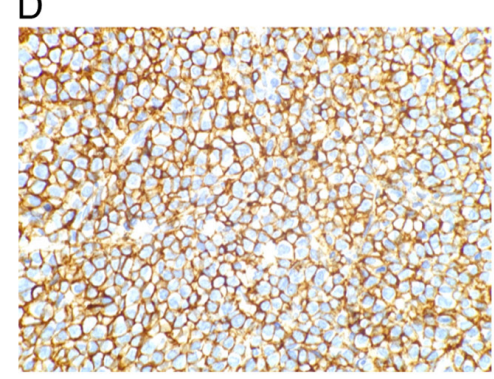

C

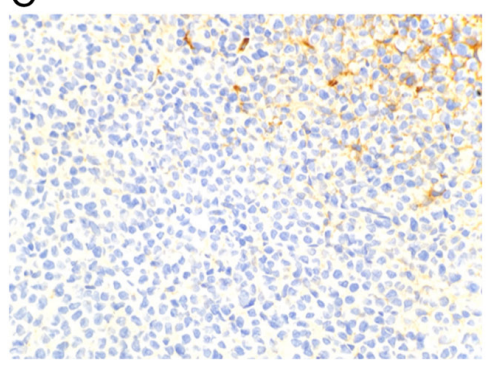

E

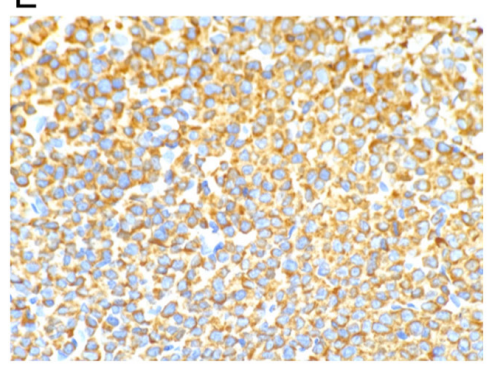

Figure 4. Bone marrow biopsy. A large number of immature plasma cells was observed on pathological examination of bone marrow biopsy, which expressed CD38 (3+), CD138 (3+), $\lambda(3+)$, and were negative for CD56 and $\kappa$. (A and B) HE staining; magnification, (A) x200 and (B) x20; (C) $\kappa$; magnification, x200; (D) CD138; magnification, x200; (E) $\lambda$; magnification, $x 200$.

for this patient according to the International Myeloma Working Group criteria, version $2014(5,6)$. Thus, bortezomib, lenalidomide and dexamethasone (BRD regimen: Bortezomib $1.3 \mathrm{mg} / \mathrm{m}^{2}$, days $1,4,8$ and 11 ; lenalidomide $25 \mathrm{mg} /$ day, days 1-21; and dexamethasone $40 \mathrm{mg} / \mathrm{day}$, days 1-4 and 8-11) were administered to this patient as the initial treatment. After 4 cycles of the BRD regimen, the multiple extramedullary lesions disappeared, serum immunofixation electrophoresis 
Table II. Antibodies used for immunohistochemistry.

\begin{tabular}{llllll}
\hline Antibody & \multicolumn{1}{c}{ Manufacturer } & \multicolumn{1}{c}{ Clone no. } & Species & Type & Platform \\
\hline CD20 & Roche Diagnostics GmbH & L26 & Mouse & Monoclonal & Ventana BenchMark XT \\
CD79a & Roche Diagnostics GmbH & SP18 & Rabbit & Monoclonal & Ventana BenchMark XT \\
CD138 & Fuzhou Maixin Biotech. Co., Ltd. & MI15 & Mouse & Monoclonal & Ventana BenchMark XT \\
$\kappa$ & Fuzhou Maixin Biotech. Co., Ltd. & RAB-0111 & Rabbit & Monoclonal & Ventana BenchMark XT \\
$\lambda$ & Fuzhou Maixin Biotech. Co., Ltd. & LAM03 + HP6054 & Mouse & Monoclonal & Ventana BenchMark XT \\
MUM1 & Fuzhou Maixin Biotech. Co., Ltd. & MUM1p & Mouse & Monoclonal & Ventana BenchMark XT \\
\hline
\end{tabular}

A

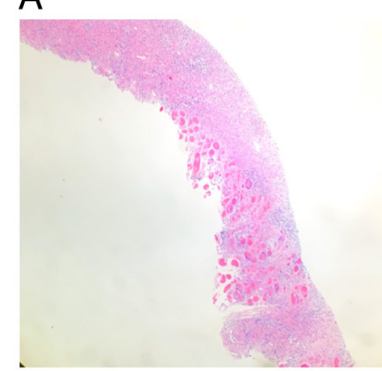

$E$
B

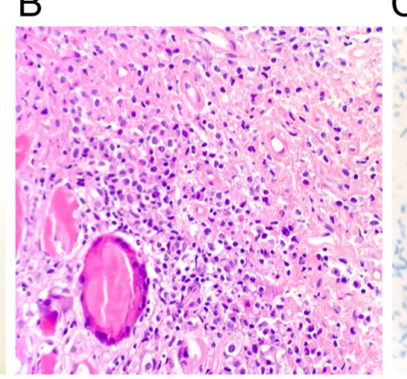

$\mathrm{F}$
C

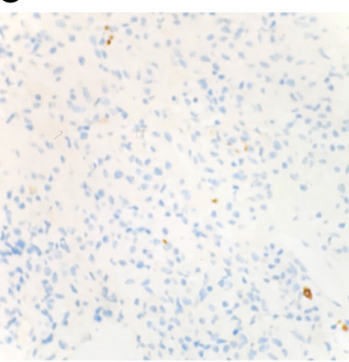

G

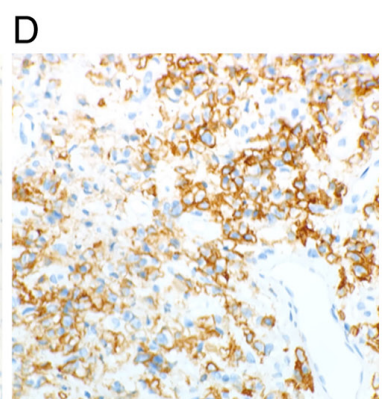

$\mathrm{H}$

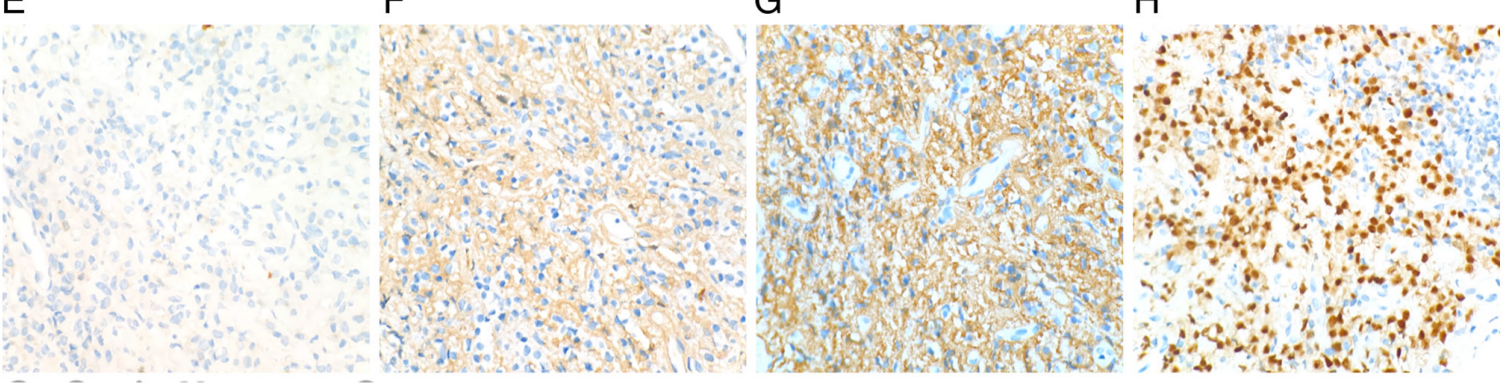

Figure 5. Chest wall mass biopsy. On pathological examination, a mass composed of immature plasma cells was found in muscular tissue. The plasma cells were positive for CD38 (3+), CD138 (3+), $\lambda(3+)$ and MUM1 (2+), and negative for CD79a, CD20 and $\kappa$. (A and B) HE staining; magnification, (A) x20 and (B) x200; (C) CD20; magnification, x200; (D) CD138; magnification, x200; (E) CD79a; magnification, x200; (F) $\kappa$; magnification, x200; (G) $\lambda$; magnification, x200; (H) MUM1; magnification, x200. MUM1, multiple myeloma oncogene 1.

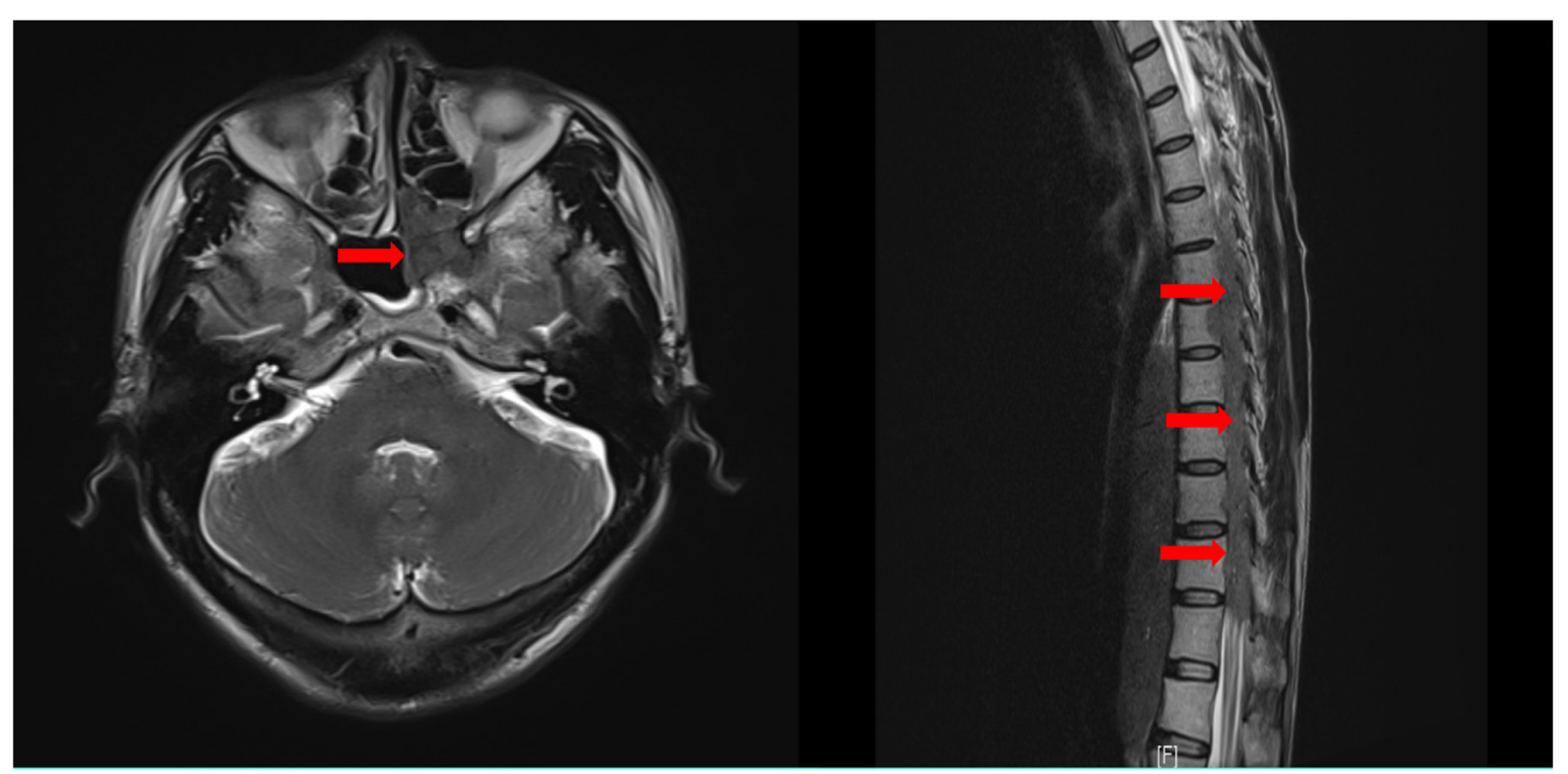

Figure 6. Enhanced magnetic resonance imaging scan of the brain and whole spine. Space-occupying lesions were identified in the sphenoid sinus and ethmoidal cellules, along with multiple foci in the T6-12 vertebral body and appendix, and T5-11 intraspinal space-occupying lesions (arrows). 
became negative, and the proportion of plasma cells in the bone marrow decreased to $1 \%$. Thus, the interim efficacy assessment was $\mathrm{CR}$, and 5 continuous cycles of the BRD regimen were administered. Considering that the patient had IgD MM with multiple extramedullary lesions at diagnosis, autologous haematopoietic stem-cell transplantation (auto-HSCT) was recommended several times during the treatment. However, the patient refused to receive auto-HSCT. After 9 cycles of the BRD regimen, the serum levels of free $\lambda$ and $\kappa$ light chains were 21.8 and $53.2 \mathrm{mg} / \mathrm{l}$, respectively. Subsequently, lenalidomide ( $25 \mathrm{mg}$ /day, days 1-21, 28 days per cycle) was administered as maintenance therapy.

At 3 months after the last chemotherapy cycle, the patient developed weakness of both lower limbs and urinary incontinence. No neurological symptoms indicating cranial nerve abnormalities were present. Contrast-enhanced MRI scans of the brain and whole spine were immediately performed, and space-occupying lesions in the sphenoid sinus and ethmoidal cellules, multiple foci the T6-12 vertebral body and appendix, and T5-11 intraspinal space-occupying lesions were identified (Fig. 6); the whole-brain contrast-enhanced MRI scan revealed no suspicious lesions. Based on the clinical history and imaging characteristics, MM involvement of the aforementioned sites was considered. The results of blood tests, biochemical detection, serum immunofixation electrophoresis and bone marrow examination were normal (Table I). Thus, taking all these findings into account, CNS involvement and extramedullary relapse were considered in this case. Subsequently, the IRD regimen, which includes ixazomib (4 $\mathrm{mg} /$ day, days 1 , 8 and 15), lenalidomide (25 mg/day, days 1-21) and dexamethasone (40 mg/day, days 1-4 and 8-11), was administered as a salvage therapy, and radiotherapy for extramedullary lesions was scheduled for this patient. Unfortunately, despite the second-line treatment strategy, the patient succumbed to the disease within 1 month after recurrence detection.

\section{Discussion}

MM is a plasma cell neoplasia characterized by diffuse tumour infiltration of the bone marrow, resulting in anaemia, bone damage with hypercalcaemia, and bone pathological fractures. Occasionally, neoplastic plasma cells acquire a different growth pattern, generating tumour masses, which is referred to as EMP. CNS involvement is a rare extramedullary manifestation of MM, with a reported incidence of $\sim 1 \%(7,8)$. MM of the CNS is strongly associated with high-risk chromosomal abnormalities, plasmablastic morphology and extramedullary manifestations $(9,10)$. Extramedullary infiltration may be identified at the time of MM diagnosis or during disease progression. However, it is more common in refractory disease or during relapse.

In recent decades, the prognosis of patients with MM has significantly improved with the introduction of immunomodulatory (IMiD) agents, proteasome inhibitors (PIs) and monoclonal antibodies $(11,12)$. Currently, PIs in combination with IMiD agents, such as those used in the BRD regimen, are considered as the first-line treatment option for active MM. However, CNS involvement remains a terminal event in most cases, with a median survival of $<6$ months (13-15). This may be attributed to the lack of effective intrathecal (IT) therapy, the limited effectiveness of radiotherapy and the limited availability of blood brain barrier (BBB)-penetrating systemic therapies (16).

Dias et al (17) performed a retrospective cohort study, enrolling 20 patients with a median follow-up of 33.5 months after CNS infiltration. The median overall survival in the group with CNS infiltration at relapse was 7.4 months, and the patients with leptomeningeal involvement had a median overall survival of 5.8 months. Varga et al (18) reported the findings from $13 \mathrm{MM}$ patients with CNS involvement, in whom the overall treatment outcome was poor; 1 patient responded to daratumumab-based treatment, whereas the effectiveness of pomalidomide and marizomib have shown some promising results.

Marizomib, a novel irreversible PI, has demonstrated promising anti-MM activity in patients with highly refractory MM (19). Previous studies have demonstrated that marizomib localizes to the CNS and significantly inhibits proteasome activity in the brain. Recent data from an ongoing phase 1 trial on malignant glioma evaluating weekly dosing demonstrated that marizomib is well tolerated and shows promising antitumour activity (20). Badros et al (21) reported the cases of 2 patients with refractory CNS-MM who benefited from marizomib-based therapy, providing additional evidence for the CNS activity of this PI and underscoring the need for further evaluation of this drug in CNS-MM.

Daratumumab is a humanized monoclonal antibody specific for CD38 that targets tumour cells via antibody-dependent cell-mediated cytotoxicity, complement-dependent cytotoxicity and phagocytosis. There are currently no available data to suggest that daratumumab can cross the BBB; however, the fact that this barrier may become more permeable in disease states and that there is no barrier in the meninges raises this possibility. Elhassadi et al (22) reported that the combined approach of craniospinal irradiation, triple IT chemotherapy and anti-CD38 monoclonal antibody produced a durable response in a MM patient with CNS involvement. Thus, the role of daratumumab in this disease status deserves further evaluation.

There were some potential limitations to this case study. First, we only had the qualitative analysis of $\operatorname{IgD}$, so we could not calculate the M protein. Second, the percentage of plasma cells was not high and the finding of CD138 positivity was not particularly clear on flow cytometry for bone marrow dilution. Third, there was no imaging evaluation for extramedullary lesions after CR. Fourth, biopsy of extramedullary foci and lumbar puncture to examine the cerebrospinal fluid were not performed at the time of relapse.

In conclusion, MM with CNS involvement is associated with extremely poor survival, and sufficient assessment of EMD is necessary during the entire course of the disease.

\section{Acknowledgements}

Not applicable.

\section{Funding}

The present study was supported by the Construction Project of Fujian Medical Center Of Hematology (grant 
no. Min201704), the Fujian Provincial Key Special Projects (grant nos. 2016Y9032, 2016B041 and 2017Y4005), the Fujian Provincial Public Health Project (grant no. WKJ2016-2-06) and the National Key Research and Development Program of China (grant no. 2016YFC0902800).

\section{Availability of data and materials}

The datasets used and/or analyzed during the current study are available from the corresponding author on reasonable request.

\section{Authors' contributions}

YC analyzed and interpreted the patient data and wrote this manuscript; YQ collected the materials; HF and JL were responsible for clinical diagnosis and treatment; LC was responsible for pathological diagnosis; SL was responsible for imaging diagnosis; TL was the chief physician responsible for this case. YC, YQ and TL have seen and can confirm the authenticity of the raw data. All the authors have read and approved the final manuscript.

\section{Ethics approval and consent to participate}

The present case report was approved by the local Ethics Committee. The patient received standard treatment for his disease, and this case report had no impact on the treatment protocol.

\section{Patient consent for publication}

The patient's son provided informed consent for the publication of this case report and any associated images.

\section{Competing interests}

The authors declare that they have no competing interests.

\section{References}

1. Palumbo A and Anderson K: Multiple myeloma. N Engl J Med 364: 1046-1060, 2011.

2. Bhutani M, Foureau DM, Atrash S, Voorhees PM and Usmani SZ: Extramedullary multiple myeloma. Leukemia 34: 1-20, 2020.

3. Sevcikova S, Minarik J, Stork M, Jelinek T, Pour L and Hajek R: Extramedullary disease in multiple myeloma-controversies and future directions. Blood Rev 36: 32-39, 2019.

4. Lu J, Lu J, Chen W, Huo Y, Huang X and Hou J; Chinese Medical Doctor Association Hematology Branch: Clinical features and treatment outcome in newly diagnosed Chinese patients with multiple myeloma: Results of a multicenter analysis. Blood Cancer J 4: e239, 2014.

5. Rajkumar SV, Dimopoulos MA, Palumbo A, Blade J, Merlini G, Mateos MV, Kumar S, Hillengass J, Kastritis E, Richardson $\mathrm{P}$, et al: International myeloma working group updated criteria for the diagnosis of multiple myeloma. Lancet Oncol 15: e538-e548, 2014.

6. Palumbo A, Avet-Loiseau H, Oliva S, Lokhorst HM, Goldschmidt H, Rosinol L, Richardson P, Caltagirone S, Lahuerta JJ, Facon T, et al: Revised International staging system for multiple myeloma: A report from International myeloma working group. J Clin Oncol 33: 2863-2869, 2015.

7. Paludo J,Painuly U, Kumar S, Gonsalves WI, Rajkumar V, Buadi F, Lacy MQ, Dispenzieri A, Kyle RA, Mauermann ML, et al: Myelomatous involvement of the central nervous system. Clin Lymphoma Myeloma Leuk 16: 644-654, 2016.
8. Fassas AB, Muwalla F, Berryman T, Benramdane R, Joseph L, Anaissie E, Sethi R, Desikan R, Siegel D, Badros A, et al: Myeloma of the central nervous system: Association with high-risk chromosomal abnormalities, plasmablastic morphology and extramedullary manifestations. Br J Haetomal 117: 103-108, 2002.

9. Fassas AB, Ward S, Muwalla F, Van Hemert R, Schluterman K, Harik S and Tricot G: Myeloma of the central nervous system: Strong association with unfavorable chromosomal abnormalities and other high-risk disease features. Leuk Lymphoma 45: 291-300, 2004.

10. Sonneveld P, Avet-Loiseau H, Lonial S, Usmani S, Siegel D, Anderson KC, Chng WJ, Moreau P, Attal M, Kyle RA, et al: Treatment of multiple myeloma with high-risk cytogenetics: A consensus of the International myeloma working group. Blood 127: 2955-2962, 2016.

11. Raza S, Safyan RA and Lentzsch S: Immunomodulatory drugs (IMiDs) in multiple myeloma. Curr Cancer Drug Targets 17: 846-857, 2017.

12. Scalzulli E, Grammatico S, Vozella F and Petrucci MT: Proteasome inhibitors for the treatment of multiple myeloma. Expert Opin Pharmacother 19: 375-386, 2018.

13. Usmani SZ, Heuck C, Mitchell A, Szymonifka J, Nair B, Hoering A, Alsayed Y, Waheed S, Haider S, Restrepo A, et al: Extramedullary disease portends poor prognosis in multiple myeloma and is over-represented in high-risk disease even in the era of novel agents. Haematologica 97: 1761-1767, 2012.

14. Katodritou E, Terpos E, Kastritis E, Delimpasis S, Symeonidis AS, Repousis P, Kyrtsonis MC, Vadikolia C, Michalis E, Polychronidou G, et al: Lack of survival improvement with novel anti-myeloma agents for patients with multiple myeloma and central nervous system involvement: The Greek myeloma study group experience. Ann Hematol 94: 2033-2042, 2015.

15. Nieuwenhuizen L and Biesma DH: Central nervous system myelomatosis: Review of the literature. Eur J Haematol 80: 1-9, 2008.

16. Egan PA, Elder PT, Deighan WI, O'Connor SJM and Alexander HD: Multiple myeloma with central nervous system relapse. Haematologica 105: 1780-1790, 2020.

17. Dias ALMS, Higashi F, Peres ALM, Cury P, Crusoé EQ and Hungria VTM: Multiple myeloma and central nervous system involvement: Experience of a Brazilian center. Rev Bras Hematol Hemoter 40: 30-36, 2018.

18. Varga G, Mikala G, Gopcsa L, Csukly Z, Kollai S, Balázs G, Botond T, Wohner N, Horváth L, Szombath G, et al: Multiple myeloma of the central nervous system: 13 cases and review of the literature. J Oncol 2018: 3970169, 2018.

19. Richardson PG, Zimmerman TM, Hofmeister CC, Talpaz M, Chanan-Khan AA, Kaufman JL, Laubach JP, Chauhan D, Jakubowiak AJ, Reich S, et al: Phase 1 study of marizomib in relapsed or relapsed and refractory multiple myeloma: NPI-0052-101 Part 1. Blood 127: 2693-2700, 2016.

20. Millward M, Price T, Townsend A, Sweeney C, Spencer A, Sukumaran S, Longenecker A, Lee L, Lay A, Sharma G, et al: Phase 1 clinical trial of the novel proteasome inhibitor marizomib with the histone deacetylase inhibitor vorinostat in patients with melanoma, pancreatic and lung cancer based on in vitro assessments of the combination. Invest New Drugs 30: 2303-2317, 2012

21. Badros A, Singh Z, Dhakal B, Kwok Y, MacLaren A, Richardson P, Trikha $\mathrm{M}$ and Hari P: Marizomib for central nervous system-multiple myeloma. Br J Haetomal 177: 221-225, 2017.

22. Elhassadi E, Murphy M, Hacking D and Farrell M: Durable treatment response of relapsing CNS plasmacytoma using intrathecal chemotherapy, radiotherapy, and Daratumumab. Clin Case Rep 6: 723-728, 2018.

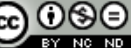

This work is licensed under a Creative Commons Attribution-NonCommercial-NoDerivatives 4.0 International (CC BY-NC-ND 4.0) License. 